\title{
Split-Ring-Resonator-Coupled Enhanced Transmission through a Single Subwavelength Aperture
}

\author{
Koray Aydin, ${ }^{1,2, *}$ A. Ozgur Cakmak, ${ }^{1}$ Levent Sahin, ${ }^{1} \mathrm{Z} . \mathrm{Li},{ }^{1}$ Filiberto Bilotti, ${ }^{3}$ Lucio Vegni, ${ }^{3}$ and Ekmel Ozbay ${ }^{1}$ \\ ${ }^{1}$ Department of Physics, Nanotechnology Research Center, and Department of Electrical and Electronics Engineering, \\ Bilkent University, 06800, Ankara, Turkey \\ ${ }^{2}$ Thomas J. Watson Laboratories of Applied Physics, California Institute of Technology, Pasadena, California 91125, USA \\ ${ }^{3}$ Department of Applied Electronics, University of Roma Tre, Rome 00146, Italy
}

(Received 26 May 2008; revised manuscript received 20 October 2008; published 7 January 2009)

We report the enhanced transmission of electromagnetic waves through a single subwavelength aperture by using a split-ring resonator (SRR) at microwave frequencies. By placing a single SRR at the near field of the aperture, strongly localized electromagnetic fields are effectively coupled to the aperture with a radius that is 20 times smaller than the resonance wavelength $(r / \lambda=0.05)$. We obtained 740-fold transmission enhancement by exciting the electric resonance of SRR. A different coupling mechanism, through the magnetic resonance of SRR, is also verified to lead to enhanced transmission.

Enhanced transmission of light through apertures that are much smaller than the wavelength has received a burgeoning amount of interest [1,2], after the seminal work by Ebbesen et al., realizing the extraordinary transmission from subwavelength hole arrays that were milled in optically thick metallic films [3]. Extraordinary light transmission has been extensively studied by using subwavelength periodic hole arrays [3-7] or metallic structures with a single aperture [7-12]. Based on Bethe's theoretical description, transmission through a single subwavelength aperture of a radius $r \ll \lambda$ scales with $(r / \lambda)^{4}$ [13]. However, one can increase the amount of light passing through a single hole by corrugating the metal surface with periodic grooves [7], filling the hole with a material of high dielectric permittivity [8,9], using alternative aperture geometries $[10,11]$, or placing artificially designed metamaterial covers in front of the aperture [12]. In all of the approaches, transmission is enhanced by a resonant process that leads to the effective coupling of light to a small aperture. Although much of the work on enhanced transmission has been carried out at optical frequencies, similar results are obtained at microwave [14] and $\mathrm{THz}$ [15] frequency regimes.

In this Letter, we propose and demonstrate an alternative approach that utilizes the resonance of a split-ring resonator (SRR) [16] in order to enhance the transmission through a single subwavelength aperture at microwave frequencies. We successfully demonstrated the extraordinary transmission of microwave radiation through an aperture of radius $r$, which is 20 times smaller than the incident wavelength $(r / \lambda=0.05)$. We measured a 740fold enhancement by using electrically coupled SRR in the proximity of a single aperture. Moreover, we showed enhanced transmission by exciting the magnetic resonance of SRRs. Enhanced transmission is attributed to the highly localized electric fields at the resonance frequency of split- ring resonators that couple incident electromagnetic (EM) radiation to the aperture.

A commercial $1.6 \mathrm{~mm}$ thick FR4 printed circuit board deposited with a thin $(30 \mu \mathrm{m})$ copper plate of the size $L \times$ $L(L=200 \mathrm{~mm})$ was used in the experiments. A circular aperture with a radius of $r=4 \mathrm{~mm}$ at the center of a metallic plate was created by mechanical etching. In the experiments, single SRR was used to couple electromagnetic waves to the aperture at a resonance frequency of SRR [Fig. 1(a)]. SRR was made up of two concentric rings
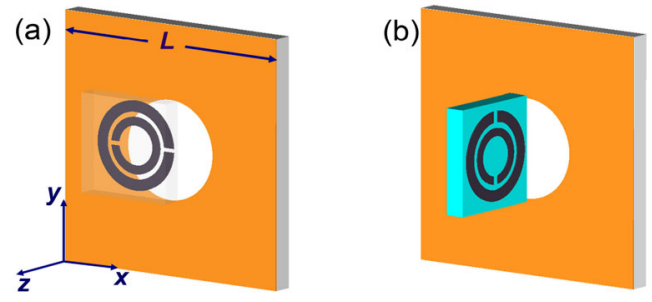

(c)

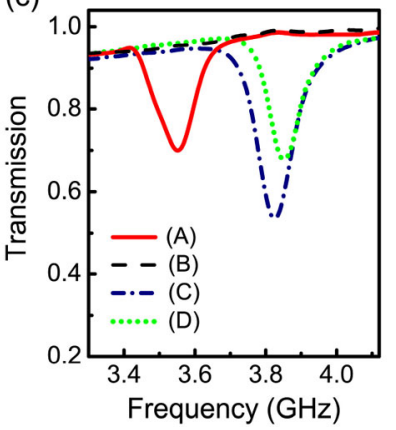

(d)

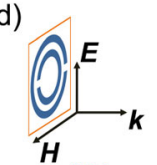

(A)

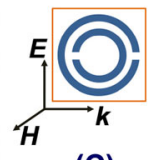

(C)

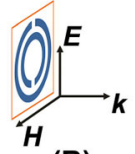

(B)

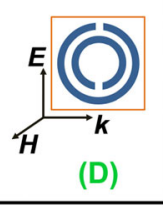

FIG. 1 (color online). Schematic drawings of a subwavelength aperture in a metallic plate and SRR plane (a) parallel and (b) perpendicular to the aperture plane. (c) Measured transmission spectra from a single SRR with four different orientations. (d) Schematic drawing of SRR orientations with respect to the incident EM field. 
with the parameters as provided in [17]. The radius of the SRR is $3.6 \mathrm{~mm}$, which is comparable with the aperture size. Figure 1(b) shows an alternative configuration of placing SRR in front of the aperture, wherein a different resonance mechanism (magnetic resonance) plays a role in the enhanced transmission.

The resonance of a single SRR for a different configuration and the incident polarization of an electromagnetic wave were studied simply by measuring the transmission spectrum. Transmission measurements were performed by using two waveguides as a transmitter and receiver, which were connected to the Agilent N5230A network analyzer. Four different orientations [Fig. 1(d)] of SRR with respect to the incident EM wave were investigated, the results of which are shown in Fig. 1(c). For the configurations SRR (A) and SRR (B), the wave propagation was perpendicular to the plane of SRRs [see Fig. 1(d)]. Although the magnetic resonance could not be excited, it is possible to excite an electrical resonance for SRR (A), due to the asymmetry of the SRR structure with respect to the electric field [18]. The resonance frequency for SRR (A) is at $3.55 \mathrm{GHz}$. For the SRR (B) case, the transmission resonance was not observed. The magnetic resonance in SRRs could only be excited when $H$ was perpendicular to the plane of SRR as in SRR (C) and (D). Both electric and magnetic fields contribute to the resonance mechanism of SRRs for configuration (C) [18]. The resonance of SRR (D) is purely of magnetic origin since the $E$ field is symmetric with the orientation of the splits. The resonance frequencies were $3.82 \mathrm{GHz}$ for SRR (C) and $3.85 \mathrm{GHz}$ for SRR (D).

In the measurements, we employed two waveguide antennas to transmit and receive electromagnetic waves. A transmitter antenna was placed $0.2 \mathrm{~mm}$ away from the metallic plate with the aperture, and the transmitted power was collected $5 \mathrm{~cm}$ away from the structure. Wave propagation was along the $z$ axis, where $E \| y$ and $H \| x$ [Fig. 1(a)]. First, we measured transmission through the metallic plate with a single subwavelength aperture [shortdashed red line in Fig. 2(a)]. As expected from the diffraction theory, the transmission of electromagnetic waves through a small aperture was very weak [13]. We then placed a single SRR $0.1 \mathrm{~mm}$ away from the aperture with the outer split region facing the center of the aperture as shown in Fig. 1(a). The solid blue line in Fig. 2(a) plots the measured intensity of the EM wave, which propagated through the SRR (A) structure and aperture. The transmission was significantly increased when a single SRR was placed at the near field of the aperture. We observed a 740fold enhancement in the transmission at $3.55 \mathrm{GHz}$ [see inset of Fig. 2(a)]. Here, we define the enhancement as the ratio of the field intensity of a transmitted EM wave through an SRR and aperture to that through the aperture only. It is noteworthy that the maximum enhancement was obtained at the resonance frequency of SRR (A). Electrically excited resonance of SRR causes the strong localization of the electric field at the splits and gaps of an SRR structure. The resonance of SRR is responsible for the

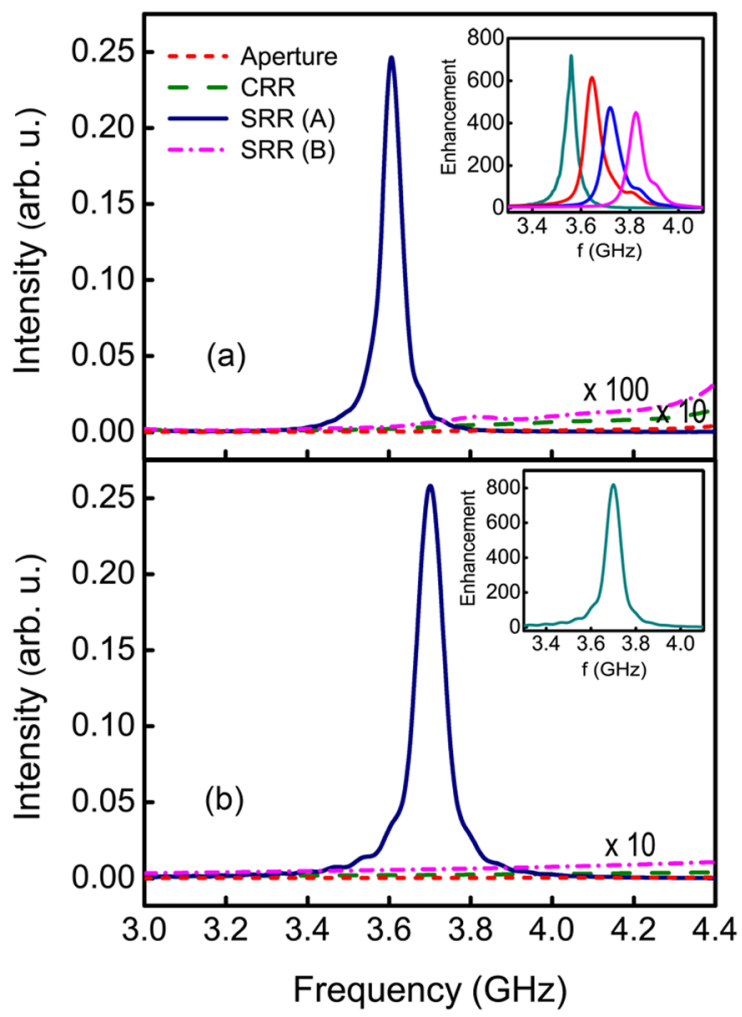

FIG. 2 (color online). (a) Measured and (b) simulated intensity of transmitted EM wave from only aperture, and aperture covered with CRR, SRR (A) and SRR (B). Insets show the enhancement factor obtained from SRR (A) and aperture. Inset of Fig. 2(a) plots enhancement factor of 3 different SRRs.

enhancement in the transmission. We performed additional measurements to verify this evidence. We placed a closed ring resonator $(\mathrm{CRR})$ in front of the aperture. CRR is comprised of two concentric rings without splits [17]. Evidently, the resonance behavior was no longer present for CRR, and the transmission was comparable with that of the aperture. We also checked the SRR (B) configuration together with the aperture, for which $E \| x$ and $H \| y$. Since the resonant coupling of EM waves to the aperture is not present for this configuration, transmission is not enhanced through the aperture. In our approach, where we utilized a bianisotropic SRR, the enhancement of microwave radiation depends on the polarization of the incident EM wave. It has been previously shown that apertures with rectangular [10,11] and elliptical [6] shapes yield enhanced transmission for a specific polarization of an incident EM wave. Here, we achieved strong polarization dependence of enhanced transmission through a circular aperture by using a bianisotropic SRR structure. The inset of Fig. 2(a) displays the transmission enhancement factors for four different SRRs having different resonance frequencies. These results show that the frequency of enhanced transmission is governed by the resonance frequency of SRRs.

Numerical simulations were performed by using CST MICROWAVE STUDIO. The dielectric constant and tangent loss of the FR4 dielectric substrate were taken as $\varepsilon=3.6$ 
and $\delta=0.01$, respectively. We modeled the waveguide antennas that were used in the experiments to transmit and detect the electromagnetic waves. Open boundary conditions were applied along all the directions. Figure 2(b) shows the numerical simulation results for only aperture, CRR, SRR (A), and SRR (B) configurations. There was a good agreement between the measured and simulated results. In the simulations, the maximum transmission through SRR (A) and aperture is observed at $3.70 \mathrm{GHz}$ and the enhancement factor is calculated as 820 . The differences between the measured and simulated results were attributed to the deviation from the ideal material parameters and the sensitivity of the distance between the SRR and aperture on the transmission.

The maximum transmission enhancement was obtained when the outer split region of SRR coincided with the center of the aperture. The highest field intensity is at the outer split region of SRR due to strong localization of the incident EM wave [19]. These strong localized modes then couple to the radiative modes of the vacuum by exciting waveguide modes in the aperture [11]. By placing the outer split region at the center of the aperture, one can obtain the highest coupling efficiency to yield maximum transmission enhancement.

The incident EM wave was propagating perpendicular to the SRR; thus, the resonance is of electrical origin. One can also achieve enhanced transmission through SRR and aperture by exciting the magnetic resonance of SRR. A single SRR is placed in front of the aperture perpendicularly, as is schematically drawn in Fig. 1(b). SRR (D) is symmetric with respect to the $E$ field, whereas SRR (C) is antisymmetric. The corresponding results are plotted in Fig. 3(a) and 3(b). The orientation of SRR is shown in the figure insets. Measurements (solid line) and simulations (dashdotted line) revealed that the transmission through the subwavelength aperture was increased for both SRR configurations. SRR (D) yielded higher transmission compared to SRR (C). The magnetic field excited the

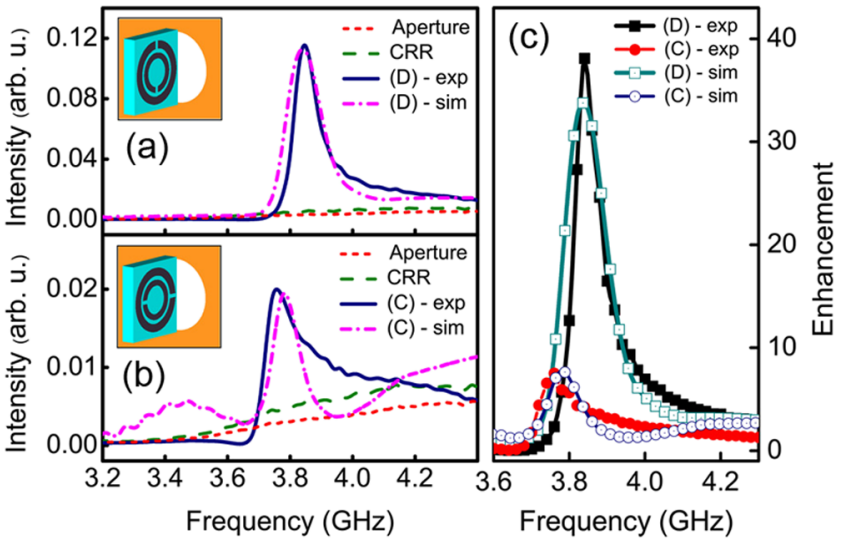

FIG. 3 (color online). Measured and simulated intensity from a single aperture covered with (a) SRR (D) and (b) SRR (C). (c) Enhancement factors obtained from the measurements and simulations for SRR (C) and (D). resonance in both orientations. However, due to the asymmetry of the SRR (C) with respect to the incident $E$ field, there occurred an electric coupling to the magnetic resonance, which was not present in SRR (D). The transmission through the aperture can be described, as a first approximation, in terms of a parallel (with respect to the screen) magnetic dipole moment and a perpendicular (with respect to the screen) electric dipole moment [13]. Because of the boundary conditions imposed by the perfect electric screen, only the aforementioned dipole moments may play a significant role. SRR (C) and SRR (D) both excite the required magnetic dipole moment; the difference is in the direction of the electric dipole moments excited across the splits. In the SRR (C) case, the electric dipole moment is parallel to the screen and, thus, does not contribute to the transmission enhancement. On the other hand, the electric dipole moment is perpendicular to the screen for the SRR (D) case, where both the magnetic and the electric dipole moments contribute to the transmission enhancement mechanism. Enhancement was measured to be 38 for configuration (D) at $3.84 \mathrm{GHz}$ and 7.5 for configuration (C) at $3.76 \mathrm{GHz}$ as plotted in Fig. 3(c). The simulation results were consistent with the experiments where we calculated the enhancement factors of 34 and 7.8 for (D) and $(\mathrm{C})$ configurations.

We calculated the amplitude of the electric field at frequencies where our simulations predicted the highest transmission. Figure 4(a) plots the $E_{y}$ amplitude evaluated at the $y=0$ plane, corresponding to the center of the structure. The amplitude of the electric field of the incident EM wave was unity. The EM wave was incident from $-z$ and propagated along the $+z$ direction. The aperture was located at $z=0$, between $x / r=-1$ and $x / r=1$. In the figures, the $x$ and $z$ values were given in terms of the radius of the aperture $r$. The solid rectangles show the positions of the dielectric FR4 substrates on which the SRR and metallic plate were deposited. The fields are localized strongly at the split regions of the SRR. The coupling of fields to the aperture via SRR is clearly seen. Figure 4(b) shows the $E_{y}$ amplitude for SRR (D) that is evaluated at the $y=3$ plane, where the outer split of the SRR is located. The incident EM wave was coupled through the aperture via the magnetic resonance of SRR. The amplitude of $E_{y}$ for the SRR (C) and aperture at the $y=0$ plane is shown in Fig. 4(c). Although the field enhancement is higher for the SRR (C) structure compared to that of SRR (D), the transmitted electromagnetic wave is apparently smaller.

In all of the experiments and simulations, the aperture radius was 20 times smaller than the wavelength $(r / \lambda=$ $0.05)$, whereas the typical aperture size utilized to achieve enhanced transmission was $r / \lambda=0.20$ [3]. In our experiments, we successfully showed that an enhanced transmission was achieved around the resonance frequency of SRR. Since the frequency of an enhanced transmission was determined by the resonance frequency of SRR, one can employ an active SRR medium [20] to tune the wavelength 


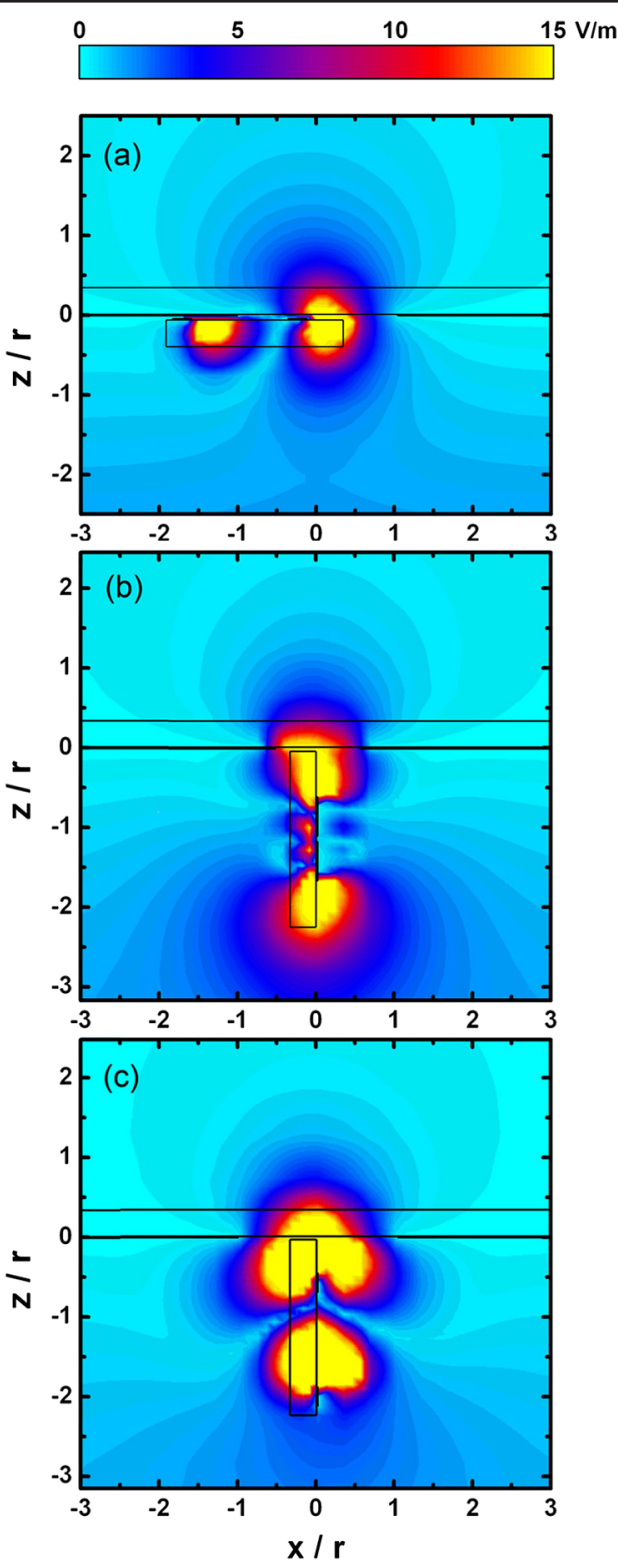

FIG. 4 (color online). Simulated $E_{y}$ magnitude for an aperture and (a) SRR (A), (b) SRR (D), and (c) SRR (C) structure.

of maximum enhancement. Utilizing SRR also provides design flexibility, in which one can easily fabricate SRRs to resonate at a desired frequency.

Our experiments were carried out at microwave frequencies, but similar results could also be obtained from $\mathrm{THz}$ [21] to near IR [22] frequencies, since SRRs have already been realized at these frequency regimes. To achieve an enhanced transmission at optical frequencies, one can fabricate an optical antenna close to a subwavelength aperture. The fields were strongly localized in the antenna feed [23], similar to the localization of fields at the SRR's split region. Note that metals are perfect conductors at micro- wave frequencies, and therefore, surface plasmons do not contribute to the enhancement process. Furthermore, surface waves were not present in our approach, since we did not use a grating structure to enhance the radiation.

To conclude, we successfully demonstrated the enhanced transmission of microwave radiation through a single subwavelength aperture by placing a single resonant element in the proximity of the aperture. The enhanced transmission was achieved by exciting the electric and/or magnetic resonance of SRR. A 740-fold enhancement was obtained through an aperture that was 20 times smaller than the wavelength. We also found that there was strong polarization dependence for enhancing the transmission, due to the bianisotropic nature of a split-ring resonator.

This work is supported by the European Union under the projects EU-METAMORPHOSE, EU-PHOREMOST, EUPHOME, and EU-ECONAM as well as TUBITAK under the Projects No. 105E066, No. 105A005, No. 106E198, and No. 106A017. One of the authors (E. O.) also acknowledges partial support from the Turkish Academy of Sciences.

*To whom all correspondence should be addressed. aydin@fen.bilkent.edu.tr

[1] C. Genet and T. W. Ebbesen, Nature (London) 445, 39 (2007).

[2] F. J. Garcia de Abajo, Rev. Mod. Phys. 79, 1267 (2007).

[3] T. W. Ebbesen et al., Nature (London) 391, 667 (1998).

[4] L. Martin-Moreno et al., Phys. Rev. Lett. 86, 1114 (2001).

[5] W. L. Barnes et al., Phys. Rev. Lett. 92, 107401 (2004).

[6] R. Gordon et al., Phys. Rev. Lett. 92, 037401 (2004).

[7] H. J. Lezec et al., Science 297, 820 (2002).

[8] F. J. Garcia de Abajo, Opt. Express 10, 1475 (2002).

[9] K. J. Webb and J. Li, Phys. Rev. B 73, 033401 (2006).

[10] A. Degiron et al., Opt. Commun. 239, 61 (2004).

[11] F. J. Garcia-Vidal et al., Phys. Rev. Lett. 95, 103901 (2005).

[12] A. Alu et al., IEEE Trans. Antennas Propag. 54, 1632 (2006).

[13] H. A. Bethe, Phys. Rev. 66, 163 (1944).

[14] S. S. Akarca-Biyikli, I. Bulu, and E. Ozbay, Appl. Phys. Lett. 85, 1098 (2004).

[15] Gomez-Rivas et al., Phys. Rev. B 68, 201306 (2003).

[16] J. B. Pendry et al., IEEE Trans. Microwave Theory Tech. 47, 2075 (1999).

[17] K. Aydin et al., Opt. Lett. 29, 2623 (2004).

[18] N. Katsarakis et al., Appl. Phys. Lett. 84, 2943 (2004).

[19] K. Aydin and E. Ozbay, J. Appl. Phys. 101, 024911 (2007).

[20] H.-T. Chen et al., Nature (London) 444, 597 (2006).

[21] T. J. Yen et al., Science 303, 1494 (2004).

[22] C. Enkrich et al., Phys. Rev. Lett. 95, 203901 (2005).

[23] P. Muhlschlegel et al., Science 308, 1607 (2005). 